\title{
Transparent Resource Management with Java RM API
}

\author{
Arkadiusz Janik and Krzysztof Zieliński \\ Institute of Computer Science, AGH, al. Mickiewicza 30, 30-059 Kraków, Poland
}

\begin{abstract}
The Multitasking Virtual Machine has been provided with many useful features like Isolation API or Resource Consumption Management API. The latter one can be used to help in managing resources in Java applications. However, using RM API does not guarantee separation between the resource management activity and the business activity. In this paper we present the concept of The Transparent Resource Management (TRM) system. The system can be used to run Java applications with resource management policies added dynamically, as a separate aspect. The policy for a given resource is dynamic which means that it may change during the runtime, depending on the state of the application, the state of the whole system, as well as the utilization of different resources in different applications.
\end{abstract}

\section{Introduction}

The new Sun's Java Multitasking Virtual Machine (2, [5]) has been provided with many interesting features like a resource consumption management ([3], 4, [7) and an isolation of Java applications ([1], 8], 6]). Although the Resource Consumption Management API provides useful features for writing resourceaware applications in Java, the application's developer using RM API has to include parts of the code responsible for managing resources directly into the application's code. In other words, the RM API provides features which can be used to build the highest-level of management policies. The main difference between the original RM API and the proposed extension is the transparency of the resource management in our solution. The RM API weakest point is the fact that much of the source code has to be written every time a new application is prepared or policy is changed. The transparency of the proposed architecture means that the resource management activity of the application is separated from its business activity and specified on the abstract level. The resource consumption management is invisible for the application. The proposed architecture makes management simple, understandable and transparent both from the application's and developer's point of view. Different management policies can be saved for later use by different developers and by different applications. The resource management functionality can be added to the target application in different cycles of the application's life and in different ways. When considering different cycles, we can add functionality either during the deployment process or during the runtime. When considering different ways of adding the 
RM, such possibilities as an aspect oriented programming ([9]) or wrappers seem to be useful. The fostered approach can be used to implement specific resource consumption management policy, to guarantee quality of service (QoS) and to add self-adaptability to Java applications ([11], [10]). In this article we present the overall, high-level architecture of The Transparent Resource Management (TRM) system. The RM API has been originally provided with Multitasking Virtual Machine; therefore presented approach bases on features of MVM.

The remainder of this paper is organized as follows. Section 2 introduces the the Resource Consumption Management API. Section 3 presents the architecture of Transparent Resource Management whereas different ways of using the Aspect Oriented Programming with the proposed architecture are discussed in Section 4. Conclusions and further research are summarized in Section 5.

\section{Overview of RM API and Isolation API}

The Resource Consumption Management API (RM API [7, 3], 4]) is provided as an experimental part of MVM. It is a framework used to supplement Java with the resource and consumption management as well as with control and reservation features. Each resource in the RM API is described as a set of resource attributes. In the RM API resource policies are encapsulated in resource domains. All isolates ([8]) that are bound to the same resource domain share the same policy. The isolate can be bound to many domains as long as each domain is responsible for a different resource. The policy is implemented by consume callbacks and triggers that specify when callbacks are called. Callbacks can act either as constraints (when called before the resource consumption is done) or as notifications (called after a consumption). An important element of the RM API is a dispenser, the bridge between the resource abstraction and its implementation. The dispenser monitors the amount of resource available for resource domains as well as it decides how much of the resource can be granted when the consumption is done. In order to stress that there is only one dispenser per resource system we should describe it as global.

The most important concept of The Application Isolation API ([] $)$ is an isolate - the abstraction of a single Java task running in a Virtual Machine. In the Sun's MVM various Java applications may be started in isolates using the same virtual machine.

\section{Transparent Resource Management Architecture}

The goal of the proposed system is to make resource management invisible from the application's point of view. The system is built on top of the RM API. What differs the TRM architecture from RM API is that in the TRM resource management is hidden from the application, not included into the application's source code and applied to it during deployment. The resource management policy is dynamic and the application's developer may specify it as a concern of the application. Dynamic means that the policy may change during the runtime, 
depending on the state of the application and the whole system. What is more, in the TRM architecture various policies may be easily applied. In contrast with the TRM architecture, the RM API can be used to build rather static policies, which are separated from the policies of different resources while in the TRM architecture the policies of different resources may be dependent. As a result, the TRM can be used to build self-adaptable applications in Java.

\subsection{High-Level Architecture}

The high-level architecture of the Transparent RM is presented in Figure 1. The architecture specifies different elements (rectangles) and different phases (arrows). The more detailed description of the elements of the system is presented below.

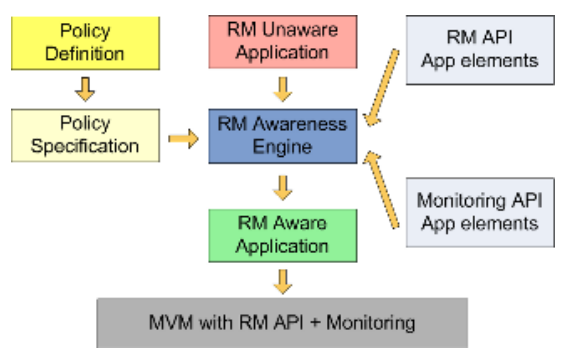

Fig. 1. The architecture of The Transparent Resource Management system

Policy definition is a high-level, user-readable description of the desired application's behavior, resource management logic and quality of service etc. This definition may limit the CPU usage or network bandwidth whereas another definition may stress that the number of concurrently running threads should not increase a given threshold. A policy definition depends on a type of the application and/or specific requirements for its current deployment. The same application running on different hardware configurations may have completely different policy definitions.

Policy specification is a formalized, more detailed presentation of the policy definition. The specialized policy specification language can be used to prepare a specification. The exemplary policy specification requires that the application should not use more than $10 \%$ of the CPU time nor to exceed $56 \mathrm{kB}$ of the network bandwidth. The limitations of different resources can be also composed to specify more complex policies like not to exceed $56 \mathrm{~kb}$ of the network bandwidth as long as CPU load is less than 90\%. If the CPU load increases, the higher network bandwidth is allowed (because of the switching off the CPU consuming socket compression module and sending raw data). The policies of different applications can be also merged as to treat previously seperate applications as a group. The exemplary policy can specify an upper limit of a number of threads that can be running concurrently in all started applications. The policy specification can 
be more or less detailed as if there was a conceptual line between the formal high-level specification and the informal low-level programming realization.

RM Unaware application is an input application which is not aware of the resource consuming policies and resource management. The developer of the application can focus on business logic. Moreover, isolating business logic from the the RM logic makes reusability possible. Each time an application is deployed/started different resource policy and different QoS can be applied.

Transformation engine is the central point of the proposed system. The engine is responsible for transforming the RM unaware application into the aware one. The transformation techniques are not presented here but will be discussed later in this paper (see Section 3.3 ). The transformation engine has four inputs:

- RM unaware application - the input application to be transformed;

- policy specification - policy to be applied to the application (presented above);

- RM API App elements - the elements of the Resource Consumption Management API to be added to the application; the transformation engine uses these elements to add (to the input application) the entity responsible for the communication with the RM API; the entity knows RM API, understands it and can use it as to enable the desired application's behavior;

- Monitoring API App elements - the elements of the Monitoring API to be added to the application; the transformation engine uses these elements to add (to the input application) the entity responsible for the communication with monitoring part of the system; the entity uses Monitoring API to provide information about the state of the whole system, as well as feedback on the application's behavior; the information from the Monitoring entity can be used by the RM entity as to modify the application's behavior and to guarantee the RM policy or the QoS;

RM Aware application is the only output from the transformation engine. The application has been modified in such a way that it can use the RM API and the Monitoring API to apply a given RM policy. More detailed description of the application after transformation is presented in Section 3.2 .

\subsection{Architecture of RM Aware Application}

An abstract view on the RM aware application (which is the result of the transformation process) is presented in Figure 2. The RM aware application is built on top of the input, the RM unaware application. Two entities have been added to the original application. There exist three types of connections between elements in the system:

- connection between entities and external environment - each entity can exchange information with the external parts of the system such as entities belonging to other applications or specialized modules common for all of the elements in the system. E.g. the monitoring entity can communicate with the monitoring module which gathers and analyzes information about the state 
of the whole system (see Figure 3). Alternatively, the monitoring entity can communicate directly with its opposite number in the different application.

- connection between entities and the RM unaware application - the connection that can be used to get information about resource utilization and/or the application's state.

- connection between different entities within the single application - this type of a connection can be used for the effective exchange of information about different aspects of the same application. As a result, the decisions made by entities are more precise.

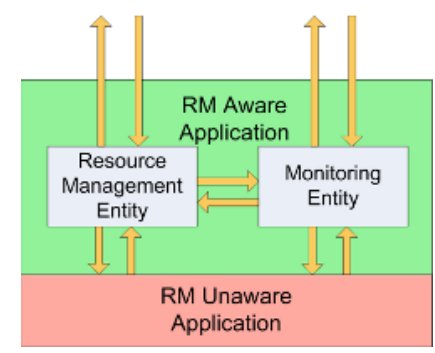

Fig. 2. The architecture of the RM aware application. The RM unaware application is instrumented with entities to communicate or juxtapose application with RM Module and Monitoring Module.

As for entities, there are a few ways of applying them. The Aspect Oriented Programming can be used to make communication between entities and original application possible.

Figure 3 illustrates four exemplary applications in the TRM system. Each application has two entities built into. Each entity communicates with the proper module (common for the whole system). The communication is bidirectional which means that modules use entities to gather information and to perform adequate actions (e.g. modify the application's behavior, definitions of RM API triggers and consume-actions etc.).

Entities of different applications can communicate with each other. This type of communication may be necessary when the strict cooperation between two applications is needed.

\subsection{Transformation Engine}

Transformation engine is the central point of the TRM system, responsible for transforming the RM unaware application into the RM aware one. The RM API is just a framework which does not imply any resource management policies. One can use it to write its own resources and resource management strategies. The desired result can be achieved by defining proper resources, resource domains and limitations (via consume-action callbacks and triggers). The obvious solution of the transformation process is to implement different resource policies by providing the specialized API (built on top of RM API) with a set of 


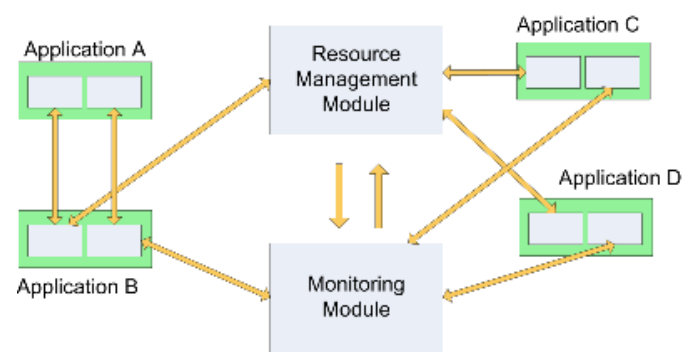

Fig. 3. Management and monitoring modules in the TRM system

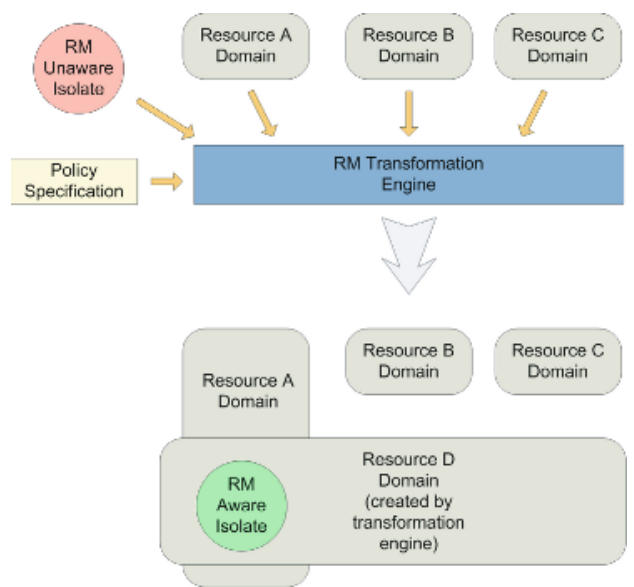

Fig. 4. The process of transforming the RM unaware isolate into the RM aware one. Resource Domain D is created by the transformation engine to meet the conditions specified in the policy specification.

ready-to-use triggers and actions. The simplest way of connecting the RM unaware application to the RM API is to use the specialized manager (isolate or program) to create and start the input application. The manager is responsible for recognition (and optionally creation) of proper resource domains, binding the original application to them and applying resource management policies (by the creation of proper triggers, actions etc.). The application's source code is not modified. However, some of the resource management limitations may result in improper behavior of the application. Let's imagine the application bound to the resource domain with limits on a number of started threads. If a maximum number of threads is present and the application wants to start another thread, resource exception is thrown and the application starts behaving in an incorrect way. The conclusion is that some code modifications might need to be done. The scheme of the transformation process is presented in Figure 4.

As it was mentioned before, the RM aware application has the resource management and monitoring entities built into. Entities can be added either by the 
use of threads "injected" into the original application or started in separated isolates. Specialized monitoring/managing modules can be used to change resource management policies dynamically (e.g. if network policy in the application A depends on CPU usage policy in the application B).

The presence of entities in the running application means that each application provides a well defined interface which can be used by other applications or by the TRM system to collect information about the application's behavior and resource utilization. Collected information may then be analyzed to check whether the conditions specified by resource policies of all applications are met. Proper activities can then be performed to guarantee that resource policy constraints are not violated. In particular, triggers and consume-action callbacks can be modified. Moreover, the architecture can be also extended to make changes of the application's behavior possible. E.g. if the CPU load increases, the application may change network traffic compression algorithm into the simplest one (less CPU usage), some of the application's features can be switched off, and some rescue features can be enabled. It is a simple way to introduce reflection and self-adaption to the RM aware applications.

\section{RM API vs Aspect Oriented Programming}

In Section 3.2 we have presented different techniques which can be used to add the resource management and monitoring modules into the RM unaware application. Aspect Oriented Programming methods can be used to support this operation.

Most applications using RM API does not see dispensers directly [3. Usually, only JRE will create and register dispensers. In this term, the user's application is unaware of the resource management. However, the resource domain's policy has to be specified as a set of pre-consume and post-consume actions or included into the trigger's code. It means that the application's developer is involved into resource management problem and has to consider it during code development.

As it is presented before, the specialized manager can be used to start the application's isolate, and bind it to proper resource domains (either the exiting or the new ones). The manager can also create and start resource managing and monitoring entities and the code responsible for changing the currently active state. The AOP can be used to let the RM unaware applications behave properly when resource exceptions are thrown. The AOP can also define jointpoints between the application and entities. The AOP may also be used to enable the self-adaptation of the running application via changing parts of the code whenever the application's state is changed.

\section{Conclusion and Future Work}

In this paper we have presented the high-level architecture of the Transparent Resource Management system. We have shown that the RM API can be used to build an additional layer which helps to separate resource management logic from 
business logic. In the proposed architecture the RM unaware application is being transformed into the RM aware one, considering a given resource policy. Different policies can be defined and stored for later use with different applications. The policies in the TRM system are dynamic, they use information about different resources in different applications. We have presented the concept of the RM and monitoring modules. The modules are used to collect the information about the state of the whole system. The information is analyzed to check whether the resource policies constraints are met. Specialized entities are used as the bridge between the system and the application. We have shown that the Aspect Oriented Programming can be used to support the process of transforming the RM unaware application into the aware one. The next goal of our research is to define the more detailed architecture of the system.

\section{References}

1. Czajkowski, G.: Application Isolation in the Java Virtual Machine. ACM OOPSLA (2000), Minneapolis, MN

2. Czajkowski, G., Daynes, L.: Multitasking without Compromise: A Virtual Machine Evolution. ACM OOPSLA (2001), Tampa, FL.

3. Czajkowski, G., Hahn, S., Skinner, G. and Soper, P., Bryce C. A Resource Management Interface for the Java Plaftorm. Sun Microsystems Laboratories Technical Raport, SMLI TR-2003-124, May 2003.

4. Czajkowski, G., Wegiel, M., Daynes, L., Palacz, K., Jordan, M., Skinner, G., Bryce, C., Resource Management for Clusters of Virtual Machines.

5. Heiss, J.: The Multi-Tasking Virtual Machine: Building a Highly Scalable JVM. Java developers forum. March 2005.

6. Czajkowski, G., Daynes, L., Wolczko, Mario., Automated and portable Native Code Isolation. April 2001.

7. Java Community Process. JSR-284: Resource Consumption Management API, http://www.jcp.org/en/jsr/detail?id=284

8. Java Community Process. JSR-121: The Application Isolation API Specification, Java Community Process, http://www.jcp.org/en/jsr/detail?id=121

9. JBoss Aspect-Oriented Programming home page. http://www.jboss.org/ products/aop

10. Sullivan, G., Aspect-Oriented Programming using Reflection and Metaobject Protocols. Arifical Intelligence Laboratory. Massachusetts Institute of Technology. April, 2001.

11. Sadjadi, S., McKinley, P., Stirewalt, R., Cheng, B., TRAP: Transparent Reflective Aspect Programming. Proceedings of the International Symposium on Distributed Objects and Applications. 2004. 